\title{
CT and SPECT image fusion using external fiducial markers for detection of the sentinel lymph nodes in breast cancer
}

\author{
Kei Haramiishi ${ }^{1}$, Shinya Nakamura ${ }^{1}$, Tomoaki Tsuchiya ${ }^{1}$, Atsushi Fukui ${ }^{1}$, Midori Matsuyama ${ }^{1}$, Mamoru Mochizuki ${ }^{1}$, \\ Kazuki Fukuchi*2 \\ ${ }^{1}$ Department of Radiology, Shizuoka General Hospital, Shizuoka, Japan \\ ${ }^{2}$ Division of Medical Technology and Science, Department of Medical Physics and Engineering, Course of Health Science, Osaka \\ University Graduate School of Medicine, Osaka, Japan
}

Received: January 30, 2017

Accepted: March 28, 2017

Online Published: April 4, 2017

DOI: $10.5430 /$ ijdi.v4n2p23

URL: https://doi.org/10.5430/ijdi.v4n2p23

\begin{abstract}
Object: Hybrid single-photon emission computed tomography/computed tomography, which is recently developed, is useful for the sentinel node (SN) mapping in patients with breast cancer. However, this expensive new technology is only available at limited hospitals. The purpose of this study was to assess the feasibility of software-based computed tomography (CT) and single-photon emission tomography (SPECT) image fusion using external fiducial markers for visualization of SNs in breast cancer.

Methods: Preoperative lymphoscintigraphy using ${ }^{99 \mathrm{~m}} \mathrm{Tc}$-phytate colloid was performed in 70 consecutive patients (mean age, $55.3 \pm 11.8$ ). Continually, SPECT and low-dose chest CT were performed using an ${ }^{241} \mathrm{Am}$-containing button as an external fiducial marker attached to the skin surface of the patient's chest wall. The acquired SPECT and CT images were rescaled, interpolated, reformatted, and registered point-by-point on a workstation.

Results: SPECT detected SN sites, including axillar $(n=96)$ and internal mammary lesions $(n=7)$. On fused images, precise overlap of hot spots shown at the corresponding lymph nodes on CT images was achieved in all but 2 cases. In cases with axillar lesions, rendering the fused images into 3D volumes with accentuation of the pectoralis minor muscle was helpful for diagnosis of SN locations in level II $(n=10)$. After surgery, all nodes were depicted as "hot nodes" on fused images, and 14 metastatic nodes were confirmed by histological examination.

Conclusions: External fiducial-based coregistration of SPECT lymphoscintigraphic and CT images depicted the precise location of SN drainage and may provide useful information for preoperative planning, without the need for hybrid SPECT/CT.
\end{abstract}

Key Words: Single-photon emission tomography, Sentinel lymph nodes, Computed tomography, Image fusion

\section{INTRODUCTION}

Presence of lymph node metastasis is a major factor in determining the stage in patients with breast cancer. ${ }^{[1]}$ It is, therefore, of clinical importance to accurately identify all sentinel nodes (SNs) before or during surgery in nonadvanced cases and SN biopsy is now accepted as part of the standard

\footnotetext{
*Correspondence: Kazuki Fukuchi, MD; Email: kfukuchi@ga2.so-net.ne.jp; Address: Division of Medical Technology and Science, Department of Medical Physics and Engineering, Course of Health Science, Osaka University Graduate School of Medicine, 1-7 Yamadaoka, Suita, Osaka 565-0871, Japan.

Published by Sciedu Press 
management of breast cancer surgery. ${ }^{[2,3]}$ For SN biopsy, lymphoscintigraphy has the potential to indicate them before surgery ${ }^{[4]}$ and can reduce morbidity relative to that of gamma probing alone. ${ }^{[5,6]}$ Although lymphoscintigraphy using planar images can identify atypical drainage patterns and identify SN sites, it provides limited spatial information because the image is acquired in only two dimensions. Thus, single-photon emission tomography (SPECT) might provide more valuable information about SN locations. In particular, SPECT has the ability to show the three-dimensional location or depth of the node and the relationships with neighboring structures. Moreover, fusion display of SPECT and chest Xray computed tomography (CT) might be the most powerful tool for indicating SNs. Recent technology advancements have led to the development of hybrid SPECT/CT. ${ }^{[7]}$ Since its introduction, hybrid SPECT/CT has been known as the most reliable imaging technique for showing the exact locations of SNs. ${ }^{[8]}$ However, this new technology is only available at a few hospitals because of the high cost of the instrumentation. Thus, software-based coregistration of SPECT and CT images is still considered to be useful and more cost effective.

The purpose of this study was to assess the feasibility of low-dose CT and SPECT image registration for visualization of SNs in breast cancer, without the use of expensive hybrid SPECT/CT.

\section{METHOD}

\subsection{Patient examinations}

This study had institutional review board approval, and all patients gave written informed consent before commencement.

On the day before surgery, SPECT of lymphoscintigraphy and low-dose multidetector CT (MDCT) were performed in 70 consecutive women with invasive breast cancer (cT0-2), with no clinical evidence of axillary lymph node metastases (cN0) and no remote metastases (cM0). The mean age of the patients was $55.3 \pm 11.8$ years.

\subsection{SPECT of lymphoscintigraphy}

A dose of $111 \mathrm{MBq}{ }^{99 m} \mathrm{Tc}$-phytate colloid was injected subcutaneously adjacent to the tumor by breast surgeons. Three hours after injection, the 3 fiducial markers were attached to the patient's skin surface at the jugular notch of the sternum and bilateral sides of the chest wall, which are located $10 \mathrm{~cm}$ below both axillas. This fiducial marker is $25 \mathrm{~mm}$ in diameter, has a 3-mm thickened plastic bottom, and contains 3.7 $\mathrm{MBq}$ of ${ }^{241} \mathrm{Am}$. The SPECT acquisition of the chest was performed by using a dual-head gamma camera (Symbia; Siemens, Knoxville, TN) and a low-energy high-resolution collimator. Thirty steps (20 sec/projection) were acquired from each head over $360^{\circ}$ of a $128 \times 128$ matrix with a pixel size of $4.8 \mathrm{~mm}$. The system was designed to operate with 2 independent energy windows, which allows acquisition of both ${ }^{99 \mathrm{~m}} \mathrm{Tc}$ and ${ }^{241}$ Am photopeaks. ${ }^{99 \mathrm{~m}} \mathrm{Tc}$ images were obtained from the photopeak (140 keV $\pm 15 \%)$, and 241 Am images were obtained from the photopeak ( $60 \mathrm{keV} \pm$ $15 \%)$. Both sets of projections were reconstructed by using the ordered subset expectation maximization algorithm (3 iterations, 1 subset) and Butterworth filtering (cutoff, 0.8 cycle/cm; order, 8).

\subsection{Low-dose CT}

After SPECT scanning was completed, the patients were moved to a CT room without detaching their radioisotopecontaining bottoms. The patients were repositioned on the CT table and maintained the same arm position of SPECT acquisition. The CT instrument was a 16-slice MDCT scanner (Brightspeed Elite, GE Healthcare). The following CT technical parameters were used: $120-\mathrm{kV}$ peak energy, 30-80 $\mathrm{mA}$ tube electric current with automated radiation exposure control, and 3.75-mm reconstruction thickness.

\subsection{Image processing and registration}

A schematic outline of the processing is shown in Figure 1. Both SPECT and CT data sets were transferred to a workstation (Ziostation2; Ziosoft Co., Tokyo, Japan), and images were altered to give the same pixel size $(4.8 \mathrm{~mm})$ and resliced using a matrix size of $128 \times 128$. Then, CT data were used as the reference, and the ${ }^{241}$ Am images were registered in three dimensions to the chest CT by using fiducial markers that enabled identification of ${ }^{241} \mathrm{Am}$ images and CT images. Process of registration were done semiautomatically but if there were some gap in the two images, the SPECT images were shifted cranio-caudally, left-right to match the CT images manually. ${ }^{[9]}$ Consequently, ${ }^{99 \mathrm{~m}} \mathrm{Tc}$ images were registered to the CT images because the ${ }^{241}$ Am images and ${ }^{99 \mathrm{~m}} \mathrm{Tc}$ images data were acquired simultaneously, so their positions were identical. The operation of registration and image fusion was performed by nuclear medicine technologists who were familiar with workstation processing. The fused SPECT and CT images were also viewed by using two-dimensional orthogonal re-slicing in axial, sagittal, and coronal orientations. Volume-rendered images were generated to localize SNs in relation to anatomical structures.

\subsection{Image analysis and evaluation}

Finally, all SPECT, CT, and fused scans were reviewed by a consensus of 2 experienced Japanese board-certified nuclear medicine physicians (10 and 24 years of experience, respectively) who also had Japanese Board of Radiology cer- 
tifications and were blinded to any information about clinical and radiologic data. All positive SNs on each image were noted and described. The SN locations were categorized as axillar (level I, II, III) and internal mammary lymphatic basins. ${ }^{[10]}$

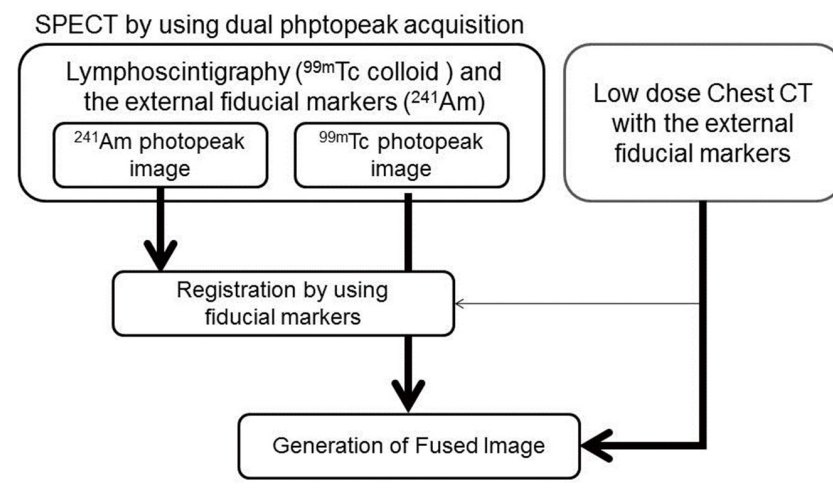

Figure 1. Flowchart of software-processing steps to produce 3D image fusion of SPECT images of lymphoscintigraphy with low-dose chest CT images

The accuracy of image coregistration of SN uptake on SPECT images and lymph nodes on CT images were scored by using
2 categories (correct or fail). If the center of the $\mathrm{SN}$ uptake deviated from the outer contour of the lymph node on the CT image, the registration was evaluated as a failure; otherwise, registration was judged to be correct.

Intraoperative gamma probe detection was used as the standard of reference. The number and the status of SNs were recorded to identify the hot $\mathrm{SNs}$ visualized rate and the concordance rate of SPECT, SPECT/CT fusion and gamma probe detection. When evaluating methods for location of hot SNs on SPECT, differentiation between level I and II was highlighted. All SNs were assessed whether having metastasis or not by routine pathology.

\subsection{Statistical analysis}

Data was entered into MS excel and statistically analyzed by VassarStats software. The diagnostic performance was expressed in terms of the sensitivity, specificity with $95 \%$ confidence intervals and the sensitivities and specificities of both SPECT alone and fuged image were calculated with intraoperative gamma probe as the gold standard. The differences in the results between them were analyzed statistically by using Fisher's exact test. $P$ values $<.05$ were considered to indicate statistical significance.
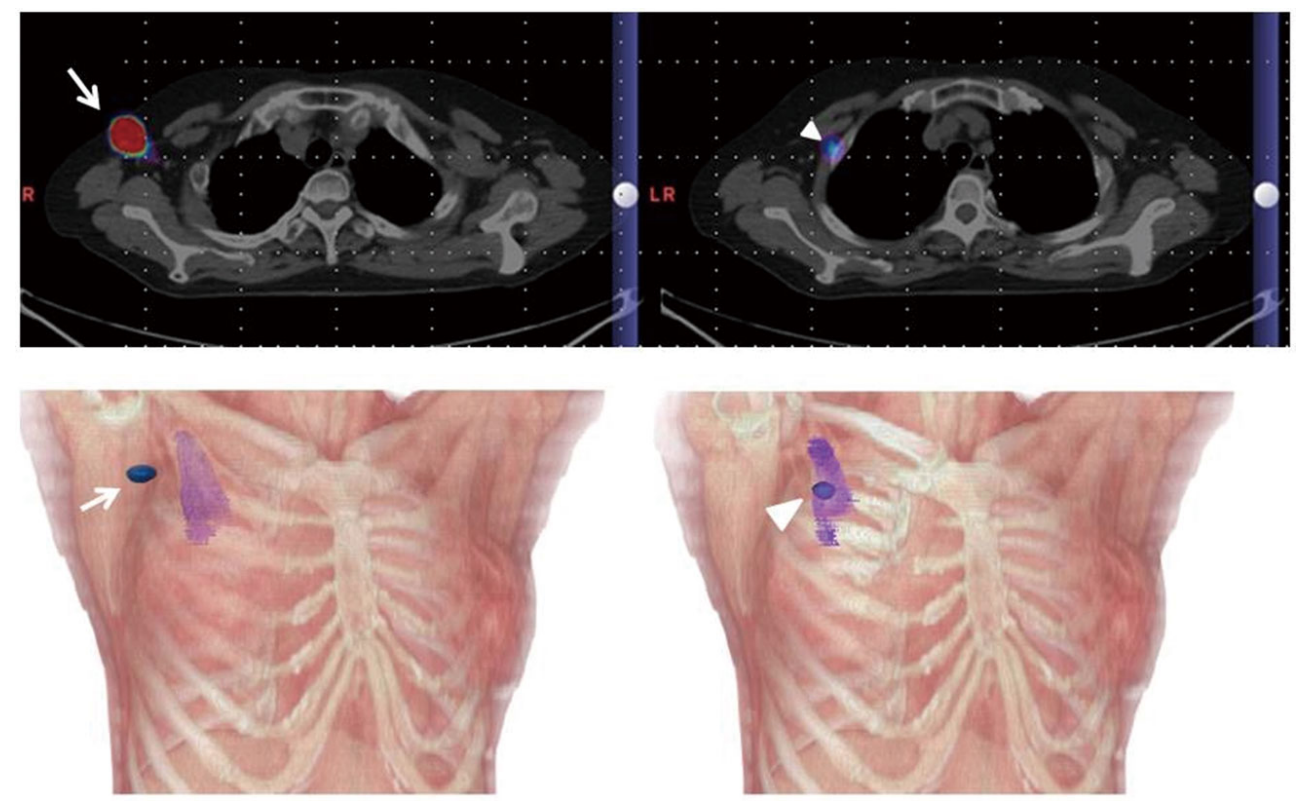

Figure 2. Typical images from a 59-year-old woman with right breast cancer. Fused SPECT and CT images depicting 2 uptake regions of ${ }^{99 \mathrm{~m}} \mathrm{Tc}$-colloid in the left axillar basins (upper panel). Three-dimensional volume-rendering $\mathrm{CT}$ images, which highlight the pectoralis minor muscle (purple colored), and SPECT fusion images clearly show drainage of ${ }^{99 m}$ Tc-colloid to the left level I (white arrow) and II (white arrowhead) axillar basins (lower panel)

\section{Results}

One hundred and five SN sites were detected by SPECT alone among all patients. There were 98 axillar lesions as- sessed as level I $(n=84)$ and II $(n=14)$ by SPECT alone. On the fused images, precise overlap of hot spots in the axillar basins $(n=96)$ of the corresponding lymph nodes on the CT 
images was achieved in all but 2 patients. The fused images depicted hot spots in the internal mammary basins $(n=7)$ for LNs that were not swollen on the CT images. In the patients with axillar lesions, rendering of the fused images into 3D volumes with accentuation of the pectoralis minor muscle was helpful for differentiation between level I $(n=86)$ and II ( $=10)$ SN locations (see Figure 2$)$. The sensitivity and specificity of SPECT only were $93 \%$ and $80 \%$, respectively but those of fused images were $97 \%$ and $100 \%$, respectively. There was a significant difference in the diagnostic performance for identifying SN locations between SPECT only and fused images $(P<.01)$.

After surgery, 14 metastatic nodes (13.6\%) were confirmed by histological examination, and all nodes were depicted as "hot nodes" on the fused images

\section{DisCussion}

In this study, we proposed an inexpensive method that uses external radioactive fiducials for coregistration and fusion of separately acquired SPECT and CT images. In our protocol, we only use three small ${ }^{241} \mathrm{Am}$-containing buttons for image coregistration. The success rate of image coregistration was quite high and only failed in two cases. Most hospitals have gamma cameras that are capable of SPECT and diagnostic X-ray CT separately, so this technique might be suitable for routine examinations without any major difficulties.

The resulting fused SPECT and CT images can depict SNs in an anatomical landscape and provide a helpful roadmap for breast surgeons. Planar lymphoscintigraphic images can show the direction of its placement and roughly estimate the depth by performing an additional orthogonal scan, but it is hard to determine the distance from the skin to the deeper SNs. SPECT/CT can provide information on the anatomical context and depth of SNs more clearly. Multidetector CT is capable of producing high-resolution 3D-volume datasets that allow a multitude of viewing formats and more complex images, such as external and internal 3D renderings that allow surgeons to virtually observe anatomical structures. In this study, we proposed viewing $\mathrm{SN}$ uptake on 3D CT images, which colors and highlights the pectoralis minor muscle. The lateral border of this muscle is a landmark for distinguishing between level I and II SN locations. This information is important for breast surgeons who perform SN biopsies. In particular, advance knowledge of the depth of SNs and surrounding structures would enable surgeons to avoid unnecessary tissue destruction during operations. ${ }^{[11]}$ Thus, 3D-rendered fused images appear to have potential for presurgical planning of SN lymphadenectomies. Especially, highlighting of the pectoralis minor muscle can be useful.

There were some limitations in our study. First, misalignment of image fusion occurred in 2 cases $(2.9 \%)$. In both cases, the SNs were located at the lateral region of level I on the CT image, and the fused image depicted hot nodes on the muscle structure. This tendency indicated that the identical body position could not be reproduced on the SPECT and CT images, which occurred because the patients could not hold their arms at the same position in both examinations. We thought combination of external fiducial markers and an individualized vacuum mattress woul be effective to avoid such misregistration. Further investigations are necessary to add for maintaining the same arm position in both SPECT and CT. ${ }^{[12]}$ Second, we estimated that there was an additional radiation exposure of approximately $2.8 \mathrm{mSv}$ per session ( $0.5 \mathrm{mSv}$ of ${ }^{241} \mathrm{Am}$ for $30 \mathrm{~min}$ during equipping and approximately $2.3 \mathrm{mSv}$ from low-dose CT acquisition). This additional exposure was estimated to be approximately $1 / 10$ of the exposure during routine diagnostic CT scanning of the chest and abdomen. For SN mapping only, a fully clinical CT study is not necessary. Thus, this level of radiation exposure might be a permissible dose for patients. Another limitation is the extra processing time required for image registration, which takes approximately 30 minutes by well-trained technicians. Nevertheless, information about the 3D distribution of the SNs does not appear to be obtainable from any other examination, so this approach should be incorporated into a clinical workflow.

\section{Conclusion}

External radioactive fiducial-based coregistration of SPECT images of lymphoscintigraphy and CT images depicted the precise location of SN drainage and provided useful information for preoperative planning, without the use of hybrid SPECT/CT, which is an advantage because not all institutions can afford SPECT/CT instrumentation.

\section{ACKNOWLEDGEMENTS}

The authors thank Kazuhiko Nakagami, MD., Michiko Tsuneizumi, MD. and Hiroyuki Takayanagi, MD. of the Department of Breast Surgery, Shizuoka General Hospital for their useful suggestions and Sumika Harakawa for her secretary support.

\section{CONFLICTS OF INTEREST DisClOSURE}

The authors have declared no conflicts of interest. 


\section{REFERENCES}

[1] Wen J, Yang Y, Liu P, et al. Development and validation of a nomogram for predicting survival on the base of modified lymph node ratio in breast cancer patients. Breast. 2017; 33: 14-22. PMid:28259045 https ://doi.org/10.1016/j.breast. 2017.01.017

[2] Vidal-Sicart S, Valdés Olmos R. Sentinel node mapping for breast cancer: current situation. J Oncol. 2012; 2012: 361341. PMid:22927845 https://doi .org/10.1155/2012/361341

[3] Lyman GH, Temin S, Edge SB, et al. Sentinel lymph node biopsy for patients with early-stage breast cancer: American Society of Clinical Oncology clinical practice guideline update. J Clin Oncol. 2014; 32: 1365-83. PMid:24663048 https://doi.org/10.1200/JCO. 2013.54.1177

[4] Goyal A, Newcombe RG, Mansel RE, et al. Role of routine preoperative lymphoscintigraphy in sentinel node biopsy for breast cancer. Eur J Cancer. 2005; 41: 238-43. PMid:15661548 https : //doi.org/10.1016/j.ejca.2004.05.008

[5] Kim SC, Kim DW, Moadel RM, et al. Using the intraoperative hand held probe without lymphoscintigraphy or using only dye correlates with higher sensory morbidity following sentinel lymph node biopsy in breast cancer: a review of the literature. World J Surg Oncol. 2005; 3: 64. PMid:16194276 https ://doi.org/10.1186/1477 $-7819-3-64$

[6] Mariani G, Moresco L, Viale G, et al. Radioguided sentinel lymph node biopsy in breast cancer surgery. J Nucl Med. 2001; 42: 1198215. PMid:11483681
[7] Lerman H, Metser U, Lievshitz G, et al. Lymphoscintigraphic sentinel node identification in patients with breast cancer: the role of SPECTCT. Eur J Nucl Med Mol Imaging. 2006; 33: 329-37. PMid:16220303 https://doi.org/10.1007/s00259-005-1927-4

[8] Vermeeren L, van der Ploeg IM, Olmos RA, et al. SPECT/CT for preoperative sentinel node localization. J Surg Oncol. 2010; 101: 184-90. PMid:19924723

[9] Samarin A, Kuhn FP, Brandsberg F, et al. Image registration accuracy of an in-house developed patient transport system for PET/CT+MR and SPECT+CT imaging. Nucl Med Commun. 2015; 36: 194-200. PMid:25321155 https://doi.org/10.1097/MNM.0000000000 000229

[10] Suami H, Pan WR, Mann GB, et al. The lymphatic anatomy of the breast and its implications for sentinel lymph node biopsy: a human cadaver study. Ann Surg Oncol. 2008; 15: 863-71. PMid:18043970 https://doi.org/10.1245/s10434-007-9709-9

[11] Uren RF, Howman-Giles R, Chung DK, et al. SPECT/CT scans allow precise anatomical location of sentinel lymph nodes in breast cancer and redefine lymphatic drainage from the breast to the axilla. Breast. 2012; 21: 480-6. PMid:22153573 https ://doi.org/10.1016/j . breast. 2011.11 .007

[12] Nakamoto Y, Sakamoto S, Okada T, et al. Clinical value of manual fusion of PET and CT images in patients with suspected recurrent colorectal cancer. AJR Am J Roentgenol. 2007; 188: 257-67. PMid:17179375 https ://doi.org/10.2214/AJR.05.0708 